\title{
Investigation of the Interfacial Electron Transfer Kinetics in Ferrocene-Terminated Oligophenyleneimine Self-Assembled Monolayers
}

\author{
Davood Taherinia $*, \uparrow+$
}

${ }^{\dagger}$ Department of Chemistry, University of Minnesota, Minneapolis, Minnesota 55455, United States

Department of Chemistry, Sharif University of Technology, Tehran 11155-9516, Iran

*Email: taherinia@ sharif.edu

Number of pages: 9

Number of figures: 3

Number of tables: 1 


\section{Calculating the Standard Rate Constants for ET}

To determine the standard ET rate constants $\left(k^{0} \mathrm{~s}\right)$ from the $\mathrm{CV}$ data, we used the method described by Myland and Oldham ${ }^{1}$ for a surface-confined redox system. Considering the following redox reaction is taking place at an electrode of area $A$ when the electrode is initially covered by a SAM of the electroactive substrate $\mathrm{S}$ with a surface concentration of $\Gamma$ :

$$
\underset{\mathrm{S}(\mathrm{ads}) \underset{k_{b}}{\stackrel{k_{f}}{\rightleftarrows}} \mathrm{P}(\mathrm{ads})+\mathrm{ne}^{-}}{ }
$$

where $k_{f}$ and $k_{b}$ represent the rate constants for forward and backward reactions, respectively, and $\mathrm{P}$ represents the product. During the redox reaction, $\mathrm{S}$ molecules get replaced by those of the product $P$, such that the total surface concentration remains constant.

$$
\Gamma_{\mathrm{S}}+\Gamma_{\mathrm{P}}=\Gamma=\text { constant. }
$$

The "coverage parameter" can be defined as

$$
\Theta=\frac{\Gamma_{P}-\Gamma_{S}}{\Gamma}
$$

which increases progressively from -1 to +1 as the reaction proceeds.

The initial potential $E_{i}$ is taken as sufficiently extreme that almost no product is present at time $t$ $=0$. The potential is then swept at a rate $v$ (sweep rate), passing through standard potential $E^{0}$ and proceeding to the final potential $E_{f}$ at which all of the $\mathrm{S}$ molecules are essentially converted to the product $\mathrm{P}$ and the sweep either stops or changes direction. During the forward the potential at a given time $t$ is

$$
E(t)=E_{i}+v t, \quad 0 \leq t \leq \frac{E_{f}-E_{i}}{v}
$$


At time $t$, the faradic charge corresponding to the redox reaction can be written as:

$$
Q(t)=n F A \Gamma_{P}=\frac{n F A \Gamma}{2}[1+\Theta(t)]
$$

where $n F A \Gamma$ is the charge needed for the complete $\mathrm{S} \rightarrow \mathrm{P}$ conversion. The faradic current flowing at time $t$ can be obtained by taking the derivative of the charge with respect to time

$$
I(t)=\frac{d Q(t)}{d t}=\frac{n F A \Gamma}{2} \frac{d \Theta}{d t}=\frac{n F A \Gamma v}{2} \frac{d \Theta}{d E}
$$

Assuming that the activities of $\mathrm{S}$ and $\mathrm{P}$ adsorbates are proportional to their fractional coverages, the faradic current can also be expressed in terms of potential-dependent rate constants described in Equation S1, thus:

$$
I(E)=n F A \Gamma\left[k_{f} \frac{\Gamma_{S}}{\Gamma}-k_{b} \frac{\Gamma_{P}}{\Gamma}\right]=\frac{n F A \Gamma}{2}\left[k_{f}-k_{b}-\left(k_{f}+k_{b}\right) \Theta(E)\right]
$$

It can be immediately seen that at the standard potential $E^{0}$, where $k_{f}=k_{b}=k^{0}$, the current and the coverage parameter are related through the simple Equation S8:

$$
\frac{I^{0}}{n F A \Gamma}=-k^{0} \Theta^{0}
$$

Combining Equations S6 and S7 results in the following differential equation:

$$
v \frac{d \Theta}{d E}=\frac{d \Theta}{d t}=k_{f}-k_{b}-\left(k_{f}+k_{b}\right) \Theta
$$

The potential-dependence of the $k_{f}$ and $k_{b}$ rate constants can be described by the well-known Butler-Vomer equation:

$$
k_{f}=k^{0} \exp \left\{\frac{(1-\alpha) n F}{R T}\left[E-E^{0}\right]\right\}
$$


and

$$
k_{b}=k^{0} \exp \left\{\frac{-\alpha n F}{R T}\left[E-E^{0}\right]\right\}
$$

where $\alpha$ is the transfer coefficient (the symmetry factor) varying between 0 and 1 . Before proceeding further, two new dimensionless parameters:

$$
\kappa=\frac{4 R T k^{0}}{n F v}
$$

and

$$
\eta=\frac{n F}{2 R T}\left[E-E^{0}\right]
$$

are defined. The parameter $\kappa$ represents the ratio between the standard rate constant of the reaction and the sweep rate. The parameter $\eta$ is a dimensionless potential scale which equals zero at the standard potential $E^{0}$. 


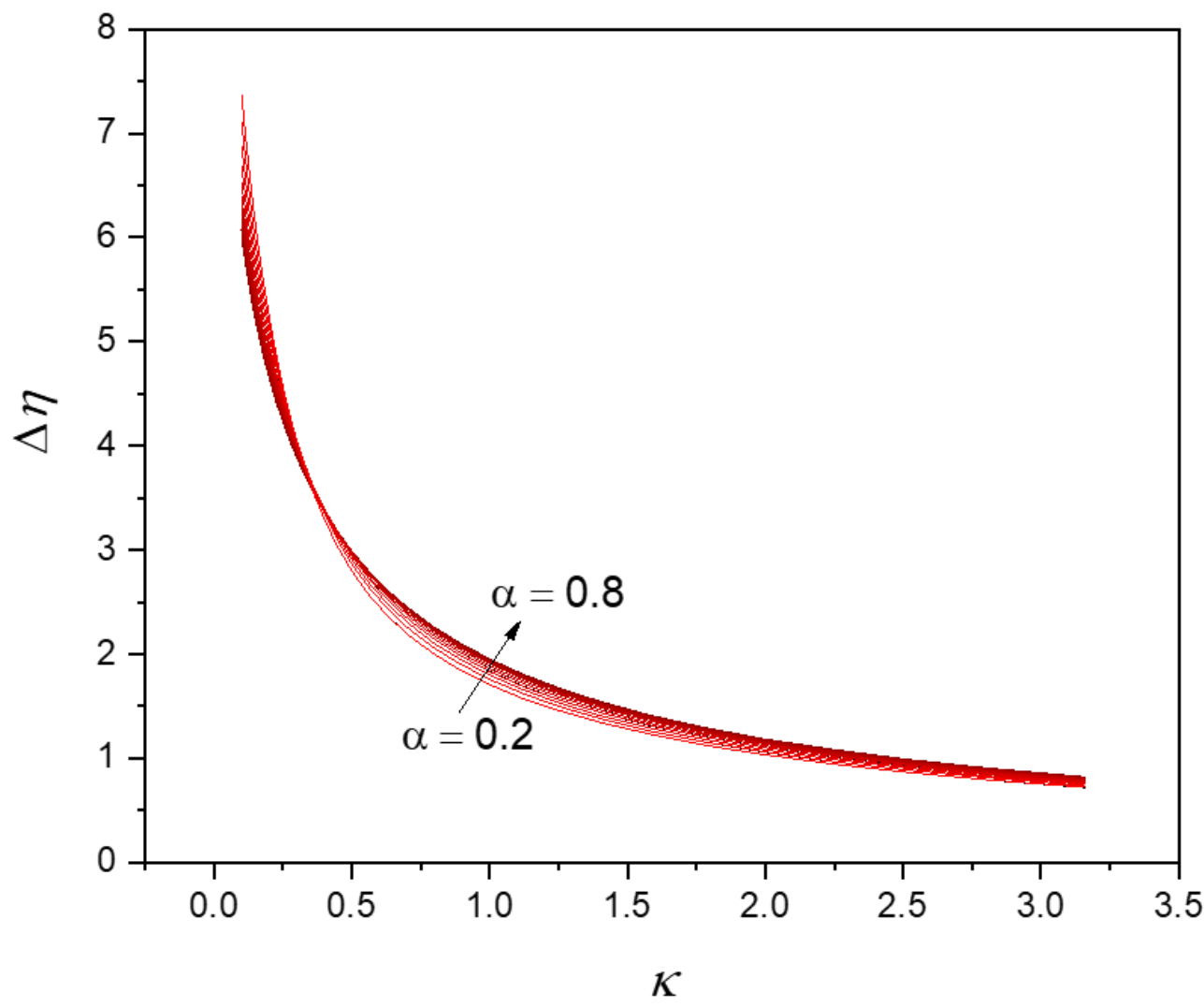

Figure S1. Plots of $\Delta \eta$ vs $\kappa$ calculated for $\alpha$ values in the range of 0.2 to 0.8 vary in the increments of 0.025 .

By plugging the Butler-Volmer expressions of $k_{f}$ and $k_{b}$ into Equation S9 and employing the new definitions, the following differential equation results:

$$
\frac{2}{\kappa} \frac{d \Theta}{d \eta}=\exp \{2(1-\alpha) \eta\}-\exp \{-2 \alpha \eta\}-[\exp \{2(1-\alpha) \eta\}+\exp \{-2 \alpha \eta\}] \Theta
$$

Equation S14 was numerically solved for different sets of $\kappa$ and $\alpha$. The solution $(\Theta(\eta))$ is essentially a CV curve from which we extracted the "dimensionless peak separation" $\Delta \eta$, defined as:

$$
\Delta \eta=\eta_{a}-\eta_{c}=\frac{n F}{2 R T}\left[E_{a}-E_{c}\right]
$$




$$
\left(\eta_{a}=\frac{n F}{2 R T}\left[E_{a}-E^{0}\right] ; \eta_{c}=\frac{n F}{2 R T}\left[E_{c}-E^{0}\right]\right)
$$

where $E_{a}$ and $E_{c}$ are anodic and cathodic peak potentials, respectively. Based on these results, plots of $\Delta \eta$ vs $\kappa$ were generated for each $\alpha$ value as depicted in Figure S1. One can see that the variation between the curves corresponding to different $\alpha$ values is relatively small and they all lie very close to each other. These plots allow us to determine the standard rate constants from the experimental CV data. To do this, we used the $\Delta \eta$ vs $\kappa$ plot for $\alpha=0.5$ and obtained the $\kappa$ (and hence the $k^{0}$ through Equation S12) corresponding to the experimental $\Delta \eta$. Figure S2 summarizes our procedure to extract the standard rate constants from the experimental $\mathrm{CV}$ data.

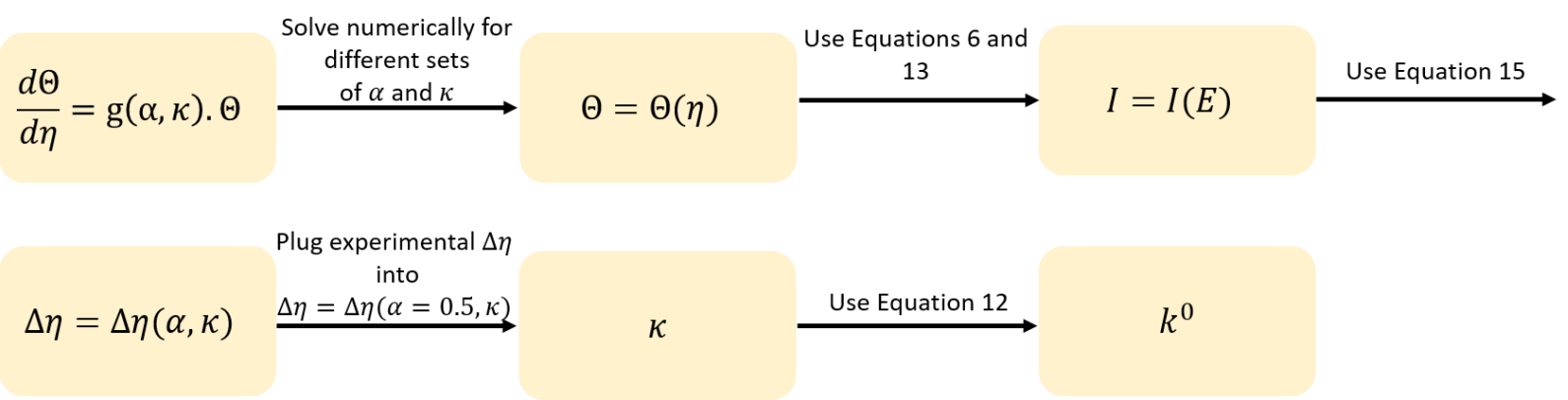

Figure S2. Flowchart representation of the procedure for determining the standard ET rate constant $\left(k^{0}\right)$ from the experimental CV peak separation.

\section{Characterization of SAMs}

Reflection-absorption infrared spectroscopy. SAM growth was monitored by reflectionabsorption infrared spectroscopy (RAIRS). OPI and CB-OPI SAMs have been extensively characterized by RAIRS and other methods. ${ }^{2-4}$ Here, the stepwise growth method followed by RAIRS is demonstrated for CB3-OPI 8p (Figure S3; the terminal " $p$ " implies the presence of a remaining reactive terminal amine or carboxaldehyde group). Figure S3A outlines the chemistry for growing the SAM. The first step is the adsorption of 4-aminothiophenol (4-ATP) 

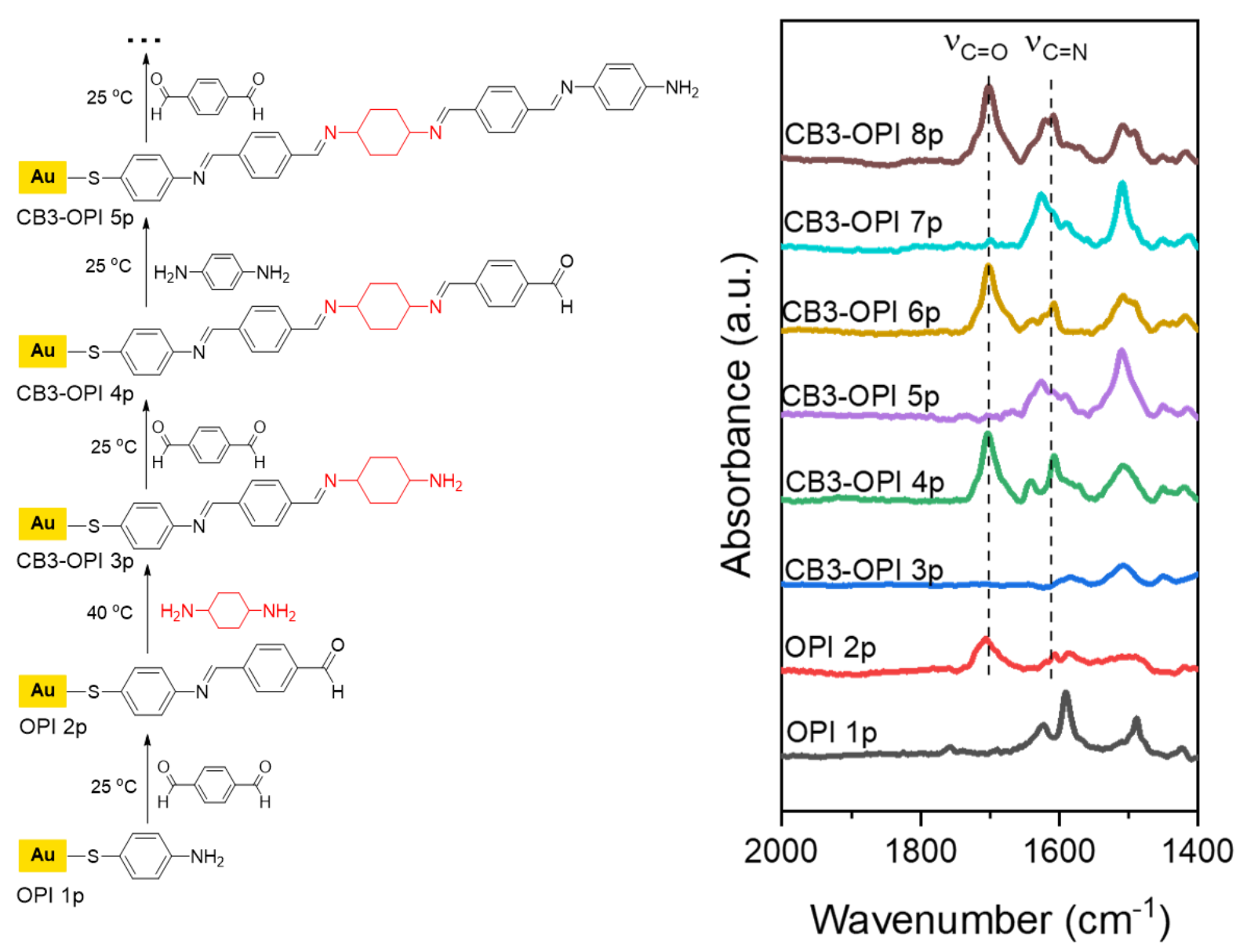

Figure S3. (A) Synthetic scheme for the stepwise growth of CB3-OPI 8p SAM on the Au substrate. (B) RAIRS spectra of the SAMs shown on the left. Dashed lines specify the positions of imine stretches $\left(\mathrm{C}=\mathrm{N}, \sim 1620 \mathrm{~cm}^{-1}\right)$, and carbonyl stretches $\left(\sim 1700 \mathrm{~cm}^{-1}\right)$.

on the Au surface. The imine bonds are then made by alternate addition of terephthalaldehyde and 1,4-diaminobenzene. Replacing the aromatic diamine with the trans-1,4-diaminocyclohexane in the growth process enabled us to incorporate a saturated spacer into the conjugated chain. The corresponding RAIRS spectra are given in Figure S3B. It can be seen that carbonyl stretches $\left(\sim 1700 \mathrm{~cm}^{-1}\right)$ alternatively appear and disappear for even and odd numbers of repeat units, respectively. This indicates that the imination reactions proceeded to near completion on the 
surface. Imine stretches $\left(\sim 1620 \mathrm{~cm}^{-1}\right)$ and aromatic ring vibrational modes $\left(\sim 1500 \mathrm{~cm}^{-1}\right)$ are also observed consistent with the growth of SAMs on the Au surface.

Ellipsometry. The thicknesses of the ferrocene-terminated SAMs were determined from the ellipsometry measurements. Estimated thicknesses were calculated by Cambridge ChemBio 3D Ultra software assuming a vertical orientation of the SAM. The molecular length was taken as the distance between terminal $\mathrm{H}$ (which belongs to the ferrocene unit) and $\mathrm{S}$ plus the Au-S bond length. It was assumed that Au-S bond length is $2.36 \AA^{5} .^{5}$ The results are summarized in Table S1. The observed difference between the measured and estimated thicknesses (the latter assumes an upright orientation) may indicate that the SAMs are tilted as reported for OPI and CB-OPI SAMs. ${ }^{2,4}$

Table S1. Surface characterization data for the ferrocene-terminated SAMs. Surface coverages were determined from the cyclic voltammograms of the ferrocene-terminated SAMs obtained at a sweep rate of $500 \mathrm{mV} / \mathrm{s}$.

\begin{tabular}{|l|c|c|c|}
\hline Monolayer & Estimated Thickness & Measured Thickness & $\Gamma\left(\mathbf{m o l e c u l e} / \mathbf{n m}^{\mathbf{2}}\right)$ \\
\hline OPI 2_Fc & 1.9 & 1.8 & 2.3 \\
\hline OPI 4_Fc & 3.3 & 2.9 & 2.9 \\
\hline OPI 6_Fc & 4.5 & 4.0 & 2.3 \\
\hline CB3-OPI 6_Fc & 4.6 & 3.9 & 2.1 \\
\hline CB5-OPI 6_Fc & 4.6 & 3.9 & 1.7 \\
\hline CB3,5-OPI 6_Fc & 4.6 & 4.0 & 1.3 \\
\hline OPI 8_Fc & 5.9 & 5.3 & 2.2 \\
\hline
\end{tabular}

CV. Surface coverages of the ferrocene-terminated SAMs were determined from the CV data. The surface coverage $(\Gamma)$ was calculated according to Equation S16:

$$
\Gamma=Q / n F A
$$


where $Q$ is the charge injected into the SAM and obtained by integrating the area under the oxidation peak of the cyclic voltammogram, $n$ is the number of electrons involved in the electrontransfer process (in this case, $n=1$ ), $F$ is the Faraday constant, and $A$ is the surface area of the monolayer which is in contact with the electrolyte solution and is defined by the area inside the $\mathrm{O}$ ring. The results are given in Table S1.

\section{References}

(1) Myland, J. C.; Oldham, K. B. Quasireversible Cyclic Voltammetry of a Surface Confined Redox System: A Mathematical Treatment. Electrochem. commun. 2005, 7 (3), 282-287.

(2) Ho Choi, S.; Kim, B.; Frisbie, C. D. Electrical Resistance of Long Conjugated Molecular Wires. Science 2008, 320 (5882), 1482-1486.

(3) Demissie, A. T.; Haugstad, G.; Frisbie, C. D. Growth of Thin, Anisotropic, $\pi$-Conjugated Molecular Films by Stepwise "Click" Assembly of Molecular Building Blocks: Characterizing Reaction Yield, Surface Coverage, and Film Thickness versus Addition Step Number. J. Am. Chem. Soc. 2015, 137 (27), 8819-8828.

(4) Taherinia, D.; Smith, C. E.; Ghosh, S.; Odoh, S. O.; Balhorn, L.; Gagliardi, L.; Cramer, C. J.; Frisbie, C. D. Charge Transport in 4 Nm Molecular Wires with Interrupted Conjugation: Combined Experimental and Computational Evidence for Thermally Assisted Polaron Tunneling. ACS Nano 2016, 10 (4).

(5) Tour, J. M.; Tour, J. M.; Jones, L. R.; Pearson, D. L.; Lamba, J. J. S.; Burgin, T. P.; Whitesides, G. M.; Allara, D. L.; Parikh, A. N.; Atre, S. V. Self-Assembled Monolayers and Multilayers of Conjugated Thiols, $\alpha, \omega$-Dithiols, and Thioacetyl-Containing Adsorbates. Understanding Attachments between Potential Molecular Wires and Gold Surfaces. J. Am. Chem. Soc. 1995, 117 (37), 9529-9534. 